Internat. J. Math. \& Math. Sci.

Vol. 22, No. 3 (1999) 511-519

S 0161-1712〈99>22511-2

(c) Electronic Publishing House

\title{
ON A CLASS OF SINGULAR HYPERBOLIC EQUATION WITH A WEIGHTED INTEGRAL CONDITION
}

\author{
SAID MESLOUB and ABDELFATAH BOUZIANI
}

(Received 15 June 1998)

\begin{abstract}
In this paper, we study a mixed problem with a nonlocal condition for a class of second order singular hyperbolic equations. We prove the existence and uniqueness of a strong solution. The proof is based on a priori estimate and on the density of the range of the operator generated by the studied problem.
\end{abstract}

Keywords and phrases. Singular hyperbolic equation, weighted integral condition, a priori estimate, strong solution.

1991 Mathematics Subject Classification. 35L20, 35L67, 34B15.

1. Position of the problem. In the domain $Q=(0, R) \times(0, T)$, with $0<R<\infty$ and $0<T<\infty$, we consider a second order hyperbolic equation with the Bessel operator

$$
\mathscr{L} u=v_{t t}-\frac{1}{r} v_{r}-v_{r r}=\mathrm{f}(r, t), \quad(r, t) \in Q .
$$

We adjoin to the above equation the initial conditions

$$
\begin{aligned}
& \ell_{1} v=v(r, 0)=\Phi(r), \quad r \in(0, R), \\
& \ell_{2} v=v_{t}(r, 0)=\Psi(r), \quad r \in(0, R),
\end{aligned}
$$

and the boundary conditions

$$
\begin{aligned}
\int_{0}^{R} r v(r, t) d r & =m(t), \quad t \in(0, T), \\
v_{r}(R, t) & =\mu(t), \quad t \in(0, T),
\end{aligned}
$$

where $f(r, t), \Phi(r), \Psi(r), m(t)$, and $\mu(t)$ are known functions. We assume that the data satisfies the following compatibility conditions:

$$
\begin{array}{ll}
\int_{0}^{R} r \Phi(r) d r=m(0), & \Phi_{r}(R)=\mu(0), \\
\int_{0}^{R} r \Psi(r) d r=m^{\prime}(0), & \Psi_{r}(R)=\mu^{\prime}(0) .
\end{array}
$$

Problem (1.1), (1.2), and (1.3) can be viewed as a nonlocal boundary problem for a singular hyperbolic equation. This problem has not been studied previously. In the case when in equation (1.1), instead of the Bessel operator, we have the operator $\left(a(r, t) v_{r}\right)_{r}$, with the Neumann condition and a linear constrain defined by $\int_{0}^{1} v(r, t) d r$ $=0$, we refer the reader to Bouziani [2]. For other problems with integral conditions, 
we turn back to Bouziani $[3,1,4]$ and references therein.

In this paper, we prove the existence, uniqueness and continuous dependence upon the data of a strong solution of problem (1.1), (1.2), and (1.3). For this, we transform problem (1.1), (1.2), and (1.3) with inhomogeneous boundary conditions (1.3) to an equivalent problem with homogeneous conditions by introducing a new unknown function $u$ defined as follows:

$$
u(r, t)=v(r, t)-U(r, t)
$$

where

$$
U(r, t)=r\left(r-\frac{4(r-R)^{2}}{R}\right) \cdot \mu(t)+\frac{12(r-R)^{2}}{R^{4}} \cdot m(t) .
$$

Then, the problem (1.1), (1.2), and (1.3) can be formulated in the following way.

$$
\begin{gathered}
\mathscr{L} u=f(r, t)-\mathscr{L} U=\mathrm{f}(r, t), \\
\ell_{1} u=\Phi(r)-\ell_{1} U=\varphi(r), \quad \ell_{2} u=\Psi(r)-\ell_{2} U=\psi(r), \\
\int_{0}^{R} r u(r, t) d r=0, \quad u_{r}(R, t)=0 .
\end{gathered}
$$

Instead of searching for the function $v$, we search for the function $u$. So, the solution of problem (1.1), (1.2), and (1.3) is given by $v(r, t)=u(r, t)+U(r, t)$.

2. Function spaces. For the investigation of the posed problem, we need some function spaces. Let $L_{\rho}^{2}(Q)$ be the weighted $L^{2}$-space with finite norm

$$
\|u\|_{L_{\rho}^{2}(Q)}=\left(\int_{Q} r u^{2} d r d t\right)^{1 / 2}
$$

The scalar product in $L_{\rho}^{2}(Q)$ is defined by

$$
(u, w)_{L_{\rho}^{2}(Q)}=(r u, w)_{L^{2}(Q)} .
$$

Let $V_{\rho}^{1,0}(Q)$ be the Hilbert space with scalar product

$$
(u, v)_{V_{\rho}^{1,0}(Q)}=(u, w)_{L_{\rho}^{2}(Q)}+\left(u_{r}, w_{r}\right)_{L_{\rho}^{2}(Q)},
$$

and with associated norm

$$
\|u\|_{V_{\rho}^{1,0}(Q)}^{2}=\|u\|_{L_{\rho}^{2}(Q)}^{2}+\left\|u_{r}\right\|_{L_{\rho}^{2}(Q)}^{2} .
$$

Weighted function spaces on the interval $(0, R)$, such as $L_{\rho}^{2}(0, R)$ and $V_{\rho}^{1}(0, R)$, are used. Their definitions are analogous to those defined on $Q$.

The problem (1.8), (1.9), and (1.10) can be written in the following operator form:

$$
L u=\mathscr{F} .
$$

$L u=\left(\mathscr{L} u, \ell_{1} u, \ell_{2} u\right), \mathscr{F}=(f, \varphi, \psi)$. The operator $L$ acts from $E$ to $F$, where $E$ is the 
Banach space of functions $u \in L_{\rho}^{2}(Q)$, satisfying conditions (1.10), with the finite norm

$$
\|u\|_{E}^{2}=\sup _{0 \leq \tau \leq T}\left(\left\|u_{t}(\cdot, \tau)\right\|_{L_{\rho}^{2}(0, R)}^{2}+\|u(\cdot, \tau)\|_{V_{\rho}^{1}(0, R)}^{2}\right)
$$

and $F$ is the Hilbert space $L_{\rho}^{2}(Q) \times V_{\rho}^{1}(0, R) \times L_{\rho}^{2}(0, R)$, which consists of elements $\mathscr{F}_{F}=(f, \varphi, \psi)$ with finite norm

$$
\|\mathscr{F}\|_{F}^{2}=\|f\|_{L_{\rho}^{2}(Q)}^{2}+\|\psi\|_{L_{\rho}^{2}(0, R)}^{2}+\|\varphi\|_{V_{\rho}^{1}(0, R)}^{2} .
$$

The domain of definition $D(L)$ of an operator $L$ is the set of all functions $u \in L^{2}(Q)$ for which $u_{t}, u_{t t}, u_{r}, u_{t r}, u_{r r} \in L^{2}(Q)$ and satisfying conditions (1.10).

Let $\bar{L}$ be the closure of an operator $L$ with domain of definition $D(\bar{L})$.

DEFINITION. We call a strong solution of the problem (1.8), (1.9), and (1.10), the solution of the operator equation

$$
\bar{L} u=\mathscr{F} \text { for all } u \in D(\bar{L}) .
$$

\section{Energy inequality and its consequences}

THEOREM 1. For any function $u \in D(L)$, we have the energy inequality

$$
\|u\|_{E} \leq c\|L u\|_{F}
$$

where $c$ is a positive constant independent of the solution $u$.

Proof. We consider the scalar product in $L^{2}\left(Q^{T}\right)$ of the equation (1.8) and the operator

$$
M u=r u_{t}-r \mathfrak{J}_{r}^{2}\left(\rho u_{t}\right)
$$

where $Q^{\tau}=(0, R) \times(0, \tau)$ with $0 \leq \tau \leq T$, and $\mathfrak{J}_{r}^{2}\left(\rho u_{t}\right)=\int_{0}^{r} \int_{0}^{\rho} \eta u_{t} d \eta d \rho$, we get

$$
\begin{aligned}
(\mathscr{L} u, M u)_{L^{2}\left(Q^{\tau}\right)}= & -\left(u_{t t}, r \mathfrak{I}_{r}^{2}\left(\rho u_{t}\right)\right)_{L^{2}\left(Q^{\tau}\right)}+\left(u_{r r}, r \mathfrak{I}_{r}^{2}\left(\rho u_{t}\right)\right)_{L^{2}\left(Q^{\tau}\right)} \\
& +\left(u_{r}, \mathfrak{J}_{r}^{2}\left(\rho u_{t}\right)\right)_{L^{2}\left(Q^{\tau}\right)}+\left(u_{t t}, r u_{t}\right)_{L^{2}\left(Q^{\tau}\right)} \\
& -\left(u_{r r}, r u_{t}\right)_{L^{2}\left(Q^{\tau}\right)}-\left(u_{r}, u_{t}\right)_{L^{2}\left(Q^{\tau}\right)}
\end{aligned}
$$

Using conditions (1.9) and (1.10), and integrating by parts each integral term of the right-hand side of (3.3) gives

$$
\begin{aligned}
-\left(u_{t t}, r \mathfrak{J}_{r}^{2}\left(\rho u_{t}\right)\right)_{L^{2}\left(Q^{\top}\right)} & =\frac{1}{2}\left\|\mathfrak{I}_{r}\left(\rho u_{t}(\cdot, \tau)\right)\right\|_{L^{2}(0, R)}^{2}-\frac{1}{2}\left\|\mathfrak{I}_{r}(\rho \psi)\right\|_{L^{2}(0, R)}^{2} \\
\left(u_{r r}, r \mathfrak{J}_{r}^{2}\left(\rho u_{t}\right)\right)_{L^{2}\left(Q^{\tau}\right)} & =-\left(u_{r}, r \mathfrak{J}_{r}\left(\rho u_{t}\right)\right)_{L^{2}\left(Q^{\tau}\right)}-\left(u_{r}, \mathfrak{J}_{r}^{2}\left(\rho u_{t}\right)\right)_{L^{2}\left(Q^{\top}\right)} \\
\left(u_{t t}, r u_{t}\right)_{L^{2}\left(Q^{\tau}\right)} & =\frac{1}{2}\left\|u_{t}(r, \tau)\right\|_{L_{\rho}^{2}(0, R)}^{2}-\frac{1}{2}\|\psi\|_{L_{\rho}^{2}(0, R)}^{2} \\
-\left(u_{r r}, r u_{t}\right)_{L^{2}\left(Q^{\tau}\right)} & =\frac{1}{2}\left\|u_{r}(r, \tau)\right\|_{L_{\rho}^{2}(0, R)}^{2}-\frac{1}{2}\left\|\varphi_{r}\right\|_{L_{\rho}^{2}(0, R)}^{2}+\left(u_{r}, u_{t}\right)_{L^{2}(Q)}
\end{aligned}
$$


Substituting (3.4), (3.5), (3.6), and (3.7) into (3.3), we obtain

$$
\begin{aligned}
\frac{1}{2}\left\|\mathfrak{I}_{r}\left(\rho u_{t}(\cdot, \tau)\right)\right\|_{L^{2}(0, R)}^{2}+\frac{1}{2}\left\|u_{t}(\cdot, \tau)\right\|_{L_{\rho}^{2}(0, R)}^{2}+\frac{1}{2}\left\|u_{r}(\cdot, \tau)\right\|_{L_{\rho}^{2}(0, R)}^{2} \\
=\frac{1}{2}\left\|\mathfrak{J}_{r}(\rho \psi)\right\|_{L^{2}(0, R)}^{2}+\frac{1}{2}\|\psi\|_{L_{\rho}^{2}(0, R)}^{2}+\frac{1}{2}\left\|\varphi_{r}\right\|_{L_{\rho}^{2}(0, R)}^{2}+\left(u_{r}, r \mathfrak{J}_{r}\left(\rho u_{t}\right)\right)_{L^{2}\left(Q^{\top}\right)} \\
\quad+\left(\mathscr{L} u, r u_{t}\right)_{L^{2}\left(Q^{\top}\right)}-\left(\mathscr{L} u, r \mathfrak{J}_{r}^{2}\left(r u_{t}\right)\right)_{L^{2}\left(Q^{\top}\right)} .
\end{aligned}
$$

Using the Cauchy inequality, the last three terms on the right-hand side of (3.8) can be estimated as follows:

$$
\begin{aligned}
\left(u_{r}, r \mathfrak{I}_{r}\left(\rho u_{t}\right)\right)_{L^{2}\left(Q^{\top}\right)} & \leq \frac{1}{2}\left\|u_{r}\right\|_{L_{\rho}^{2}\left(Q^{\top}\right)}^{2}+\frac{R}{2}\left\|\mathfrak{I}_{r}\left(\rho u_{t}\right)\right\|_{L^{2}\left(Q^{\top}\right)}^{2}, \\
\left(\mathscr{L} u, r u_{t}\right)_{L^{2}\left(Q^{\top}\right)} & \leq \frac{1}{2}\|\mathscr{L} u\|_{L_{\rho}^{2}\left(Q^{\top}\right)}^{2}+\frac{1}{2}\left\|u_{t}\right\|_{L_{\rho}^{2}\left(Q^{\top}\right)}, \\
-\left(\mathscr{L} u, r \mathfrak{J}_{r}^{2}\left(\rho u_{t}\right)\right)_{L^{2}\left(Q^{\top}\right)} & \leq \frac{1}{2}\|\mathscr{L} u\|_{L_{\rho}^{2}\left(Q^{\top}\right)}^{2}+\frac{R^{3}}{4}\left\|\mathfrak{I}_{r}\left(\rho u_{t}\right)\right\|_{L^{2}\left(Q^{\top}\right)}^{2} .
\end{aligned}
$$

Substitution of (3.9), (3.10), and (3.11) in (3.8) gives the following inequality:

$$
\begin{aligned}
\left\|\mathfrak{J}_{r}\left(\rho u_{t}(\cdot, \tau)\right)\right\|_{L^{2}(0, R)}^{2}+\| u_{t}(\cdot, \tau) & \left\|_{L_{\rho}^{2}(0, R)}^{2}+\right\| u_{r}(\cdot, \tau) \|_{L_{\rho}^{2}(0, R)}^{2} \\
\leq & 2\|\mathscr{L} u\|_{L_{\rho}^{2}\left(Q^{\tau}\right)}^{2}+\|\varphi\|_{L_{\rho}^{2}(0, R)}^{2}+\left(\frac{R^{4}}{2}+1\right)\|\psi\|_{L_{\rho}^{2}(0, R)}^{2} \\
& +R\left(\frac{R^{2}}{2}+1\right) \cdot\left\|\mathfrak{I}_{r}\left(\rho u_{t}\right)\right\|_{L^{2}\left(Q^{\tau}\right)}^{2}+\left\|u_{t}\right\|_{L_{\rho}^{2}\left(Q^{\tau}\right)}^{2}+\left\|u_{r}\right\|_{L_{\rho}^{2}\left(Q^{\tau}\right)}^{2} .
\end{aligned}
$$

By virtue of the elementary inequality,

$$
\|u(r, \tau)\|_{L_{\rho}^{2}(0, R)}^{2} \leq\|u\|_{L_{\rho}^{2}\left(Q^{\top}\right)}^{2}+\left\|u_{t}\right\|_{L_{\rho}^{2}\left(Q^{\top}\right)}^{2}+\|\varphi\|_{L_{\rho}^{2}(0, R)}^{2},
$$

and (3.12), we have

$$
\begin{aligned}
\left\|\mathfrak{J}_{r}\left(\rho u_{t}(r, \tau)\right)\right\|_{L^{2}(0, R)}^{2}+\|u(r, \tau)\|_{V_{\rho}^{1}(0, R)}^{2}+\left\|u_{t}(r, \tau)\right\|_{L_{\rho}^{2}(0, R)}^{2} \\
\leq c_{1}\left(\|\mathscr{L} u\|_{L_{\rho}^{2}\left(Q^{\top}\right)}^{2}+\|\psi\|_{L_{\rho}^{2}(0, R)}^{2}+\|\varphi\|_{V^{1}(0, R)}^{2}\right) \\
\quad+c_{2}\left(\|u\|_{V_{\rho}^{1,0}\left(Q^{\tau}\right)}^{2}+\left\|u_{t}\right\|_{L_{\rho}^{2}\left(Q^{\tau}\right)}^{2}\right)
\end{aligned}
$$

where

$$
c_{1}=\max \left(\left(\frac{R^{4}}{2}+1\right), 2\right)
$$

and

$$
c_{2}=\max \left(1, R\left(1+\frac{R^{2}}{2}\right)\right) .
$$

Applying [2, Lem. 1], to the above inequality, we get

$$
\begin{aligned}
\| \mathfrak{I}_{r}\left(\rho u_{t}(r, \boldsymbol{\tau})\right) & \left\|_{L^{2}(0, R)}^{2}+\right\| u(r, \tau)\left\|_{V_{\rho}^{1}(0, R)}^{2}+\right\| u_{t}(r, \tau) \|_{L_{\rho}^{2}(0, R)}^{2} \\
& \leq c_{1} e^{c_{2} \tau}\left(\|f\|_{L_{\rho}^{2}\left(Q^{\tau}\right)}^{2}+\|\varphi\|_{V_{\rho}^{1}(0, R)}^{2}+\|\psi\|_{L_{\rho}^{2}(0, R)}^{2}\right) \\
& \leq c_{1} e^{c_{2} T}\left(\|f\|_{L_{\rho}^{2}(Q)}^{2}+\|\varphi\|_{V_{\rho}^{1}(0, R)}^{2}+\|\psi\|_{L_{\rho}^{2}(0, R)}^{2}\right) .
\end{aligned}
$$


Since the first term on the left-hand side of (3.17) is positive, we have

$$
\|u(r, \tau)\|_{V_{\rho}^{1}(0, R)}^{2}+\left\|u_{t}(r, \tau)\right\|_{L_{\rho}^{2}(0, R)}^{2} \leq c_{1} e^{c_{2} T}\left(\|f\|_{L_{\rho}^{2}(Q)}^{2}+\|\varphi\|_{V_{\rho}^{1}(0, R)}^{2}+\|\psi\|_{L_{\rho}^{2}(0, R)}^{2}\right) .
$$

The right-hand side of the above inequality does not depend on $\tau$. By taking the supremum with respect to $\tau$ over 0 to $T$, we get the desired inequality (3.1) with $c=c_{1}^{1 / 2} e^{c_{2} T / 2}$.

Proposition 1. The operator $L$ acting on $E$ into $F$ is closable.

Proof. Let $u_{n} \in D(L)$ be a sequence such that

$$
u_{n} \underset{n \rightarrow \infty}{\longrightarrow} 0 \text { in } E
$$

and

$$
L u_{n} \underset{n \rightarrow \infty}{\longrightarrow} \mathscr{F}=(f, \varphi, \psi) \text { in } F,
$$

we then must show that $f \equiv 0, \varphi \equiv 0, \psi \equiv 0$.

Since (3.19) holds, then we have

$$
u_{n} \underset{n \rightarrow \infty}{\longrightarrow} 0 \text { in } \mathscr{D}^{\prime}(Q),
$$

where $\mathscr{D}^{\prime}(Q)$ is the space of distributions on $Q$.

By virtue of the continuity of derivation of $\mathscr{D}^{\prime}(Q)$ in $\mathscr{D}^{\prime}(Q)$, (3.21) implies that

$$
\mathscr{L} u_{n} \underset{n \rightarrow \infty}{\longrightarrow} 0 \text { in } \mathscr{D}^{\prime}(Q) \text {. }
$$

According to (3.20), we have

$$
\mathscr{L} u_{n} \underset{n \rightarrow \infty}{\longrightarrow} f \text { in } L_{\rho}^{2}(Q) .
$$

Then

$$
\mathscr{L} u_{n} \underset{n \rightarrow \infty}{\longrightarrow} f \text { in } \mathscr{D}^{\prime}(Q) .
$$

By virtue of the uniqueness of the limit in $\mathscr{D}^{\prime}(Q)$, we conclude that $f \equiv 0$.

According to (3.20), we also conclude that

$$
\ell_{1} u_{n} \underset{n \rightarrow \infty}{\longrightarrow} \varphi \text { in } V_{\rho}^{\prime}(0, R)
$$

and that the canonical injection from $V_{\rho}^{1}(0, R)$ into $\mathscr{D}^{\prime}(0, R)$ is continuous. Hence, we deduce that

$$
\ell_{1} u_{n} \underset{n \rightarrow \infty}{\longrightarrow} \varphi \text { in } \mathscr{D}^{\prime}(0, R) .
$$

Moreover, since (3.19) holds and

$$
\left\|\ell_{1} u_{n}\right\|_{V_{\rho}^{1}(0, R)} \leq\left\|u_{n}\right\|_{E} \quad \forall n,
$$

we have

$$
\ell_{1} u_{n} \underset{n \rightarrow \infty}{\longrightarrow} 0 \text { in } V_{\rho}^{1}(0, R) .
$$

Hence, 


$$
\ell_{1} u_{n} \underset{n \rightarrow \infty}{\longrightarrow} 0 \text { in } \mathscr{D}^{\prime}(0, R) \text {. }
$$

By virtue of the uniqueness of the limit in $\mathscr{D}^{\prime}(0, R)$, we conclude, from (3.26) and (3.29), that $\varphi \equiv 0$. Using the same procedure, we can show that $\psi \equiv 0$. This proves Proposition 1.

The inequality (3.1) can be extended to strong solutions after passing to limit, that is we have

$$
\|u\|_{E} \leq c\|\bar{L} u\|_{F} \text { for all } D(\bar{L}) .
$$

The above inequality leads to the following results:

COROLLARY 1. If a strong solution of the problem (1.8), (1.9), and (1.10) exists, it is unique and depends continuously upon the data $\mathscr{F}=(f, \varphi, \psi) \in F$.

COROLLARY 2. The range $R(\bar{L})$ of the operator $\bar{L}$ is closed and equals to $\overline{R(L)}$.

\section{Existence of the solution}

THEOREM 2. For each $f \in L_{\rho}^{2}(Q), \varphi \in V_{\rho}^{1}(0, R)$, and $\psi \in L_{\rho}^{2}(0, R)$, there exists a unique strong solution $u=\bar{L}^{-1} \mathscr{F}_{F}=\overline{L^{-1}} \mathscr{F}$ of the problem (1.8), (1.9), and (1.10) satisfying the estimate

$$
\|u\|_{E} \leq c\|\mathscr{F}\|_{F}
$$

where $c$ is a positive constant independent of the solution $u$.

Proof. From the inequality (3.1), it follows that the operator $\bar{L}$ has an inverse and, from Corollary 2, we deduce that the range $R(\bar{L})$ of the operator $\bar{L}$ is closed. Hence, it suffices to prove the density of the set $R(L)$ in $F$, that is $\overline{R(L)}=F$. First, we need to prove the following proposition.

Proposition 2. If, for any $\omega \in L^{2}(Q)$ and for all $u \in D_{0}(L)=\{u \mid u \in D(L)$ : $\left.\ell_{1} u=\ell_{2} u=0\right\}$, we have

$$
(\mathscr{L} u, \omega)_{L_{\rho}^{2}(Q)}=0
$$

then $\omega$ vanishes almost everywhere in $Q$.

Proof of THe Proposition. Relation (4.2) is given for any function $u \in D_{0}(L)$, so we can express it in the following particular form:

Let $u_{t t}$ be the solution of the equation

$$
u_{t t}-\mathfrak{J}_{r}^{2}\left(\rho u_{t t}\right)=\Psi(r, t),
$$

where

$$
\Psi(r, t)=\int_{t}^{T} \omega(r, \tau) d \tau .
$$

And let the function $u$ be defined by 


$$
u=\left\{\begin{array}{l}
0, \quad \text { if } 0 \leq t \leq s, \\
\int_{s}^{t}(t-\tau) u_{\tau \tau} d \tau, \quad \text { if } s \leq t \leq T .
\end{array}\right.
$$

From relations (4.3) and (4.4), we have

$$
\omega(r, t)=\left(-u_{t t}+\mathfrak{J}_{r}^{2}\left(\rho u_{t t}\right)\right)_{t} .
$$

The function $u$ defined by relations (4.3) and (4.5) which imply that $u$ is in $D_{0}(L)$, has a high order of smoothness.

LEMmA. The function $u$ defined by (4.3) and (4.5) has derivatives with respect to $t$ up to the third order and belongs to $L_{\rho}^{2}\left(Q_{s}\right)$, where $Q_{s}=(0, R) \times(s, T)$.

Proof. For the proof, the reader should refer to [4].

To complete the proof of Proposition 2, we replace $\omega$ in (4.2) by its representation (4.6). We have

$$
\begin{aligned}
- & \left(u_{t t}, u_{t t t}\right)_{L_{\rho}^{2}\left(Q_{s}\right)}+\left(u_{t t}, \mathfrak{J}_{r}^{2}\left(\rho u_{t t t}\right)\right)_{L_{\rho}^{2}\left(Q_{s}\right)}+\left(u_{r}, u_{t t t}\right)_{L^{2}\left(Q_{s}\right)} \\
& -\left(u_{r}, \mathfrak{J}_{r}^{2}\left(\rho u_{t t t}\right)\right)_{L^{2}\left(Q_{s}\right)}+\left(u_{r r}, u_{t t t}\right)_{L_{\rho}^{2}\left(Q_{s}\right)}-\left(u_{r r}, \mathfrak{J}_{r}^{2}\left(\rho u_{t t t}\right)\right)_{L_{\rho}^{2}\left(Q_{s}\right)}=0 .
\end{aligned}
$$

Conditions (1.10), the special form of $u$ given by relations (4.3) and (4.5), and an integration by parts for each term, give

$$
\begin{aligned}
-\left(u_{t t}, u_{t t t}\right)_{L_{\rho}^{2}\left(Q_{s}\right)} & =\frac{1}{2}\left\|u_{t t}(r, s)\right\|_{L^{2}(0, R)}^{2}, \\
\left(u_{t t}, \mathfrak{J}_{r}^{2}\left(\rho u_{t t t}\right)\right)_{L_{\rho}^{2}\left(Q_{s}\right)} & =\frac{1}{2}\left\|\mathfrak{J}_{r}^{2}\left(\rho u_{t t}(\rho, s)\right)\right\|_{L^{2}(0, R)}^{2}, \\
\left(u_{r}, u_{t t t}\right)_{L^{2}\left(Q_{s}\right)} & =-\left(u_{r t}, u_{t t}\right)_{L^{2}\left(Q_{s}\right)}, \\
\left(u_{r r}, u_{t t t}\right)_{L_{\rho}^{2}\left(Q_{s}\right)} & =\frac{1}{2}\left\|u_{r t}(r, T)\right\|_{L_{\rho}^{2}(0, R)}^{2}+\left(u_{r t}, u_{t t}\right)_{L^{2}\left(Q_{s}\right)}, \\
-\left(u_{r r}, \mathfrak{J}_{r}^{2}\left(\rho u_{t t t}\right)\right)_{L_{\rho}^{2}\left(Q_{s}\right)} & =-\left(u_{r t}, \mathfrak{J}_{r}\left(\rho u_{t t}\right)\right)_{L_{\rho}^{2}\left(Q_{s}\right)}+\left(u_{r}, \mathfrak{J}_{r}^{2}\left(\rho u_{t t t}\right)\right)_{L^{2}\left(Q_{s}\right)} .
\end{aligned}
$$

Substituting (4.8), (4.9), (4.10), (4.11), and (4.12) into (4.7), we get

$$
\begin{aligned}
\left\|u_{t t}(r, s)\right\|_{L_{\rho}^{2}(0, R)}^{2} & +\left\|u_{r t}(r, T)\right\|_{L^{2}(0, R)}^{2}+\left\|\mathfrak{I}_{r}\left(\rho u_{t t}(\rho, s)\right)\right\|_{L^{2}(0, R)}^{2} \\
& =2\left(u_{r t}, \mathfrak{J}_{r}\left(\rho u_{t t}\right)\right)_{L_{\rho}^{2}\left(Q_{s}\right)} .
\end{aligned}
$$

Using the Cauchy inequality, the right member of (4.13) can be bounded and results

$$
\begin{aligned}
\left\|u_{t t}(r, s)\right\|_{L_{\rho}^{2}(0, R)}^{2} & +\left\|u_{r t}(r, T)\right\|_{L^{2}(0, R)}^{2}+\left\|\left(\mathfrak{J}_{r}\left(\rho u_{t t}(r, s)\right)\right)\right\|_{L^{2}(0, R)}^{2} \\
& \leq R\left\|u_{r t}\right\|_{L^{2}\left(Q_{s}\right)}^{2}+R\left\|\mathfrak{J}_{r}\left(\rho u_{t t}\right)\right\|_{L^{2}\left(Q_{s}\right)}^{2} .
\end{aligned}
$$

We observe that the integrand in the second member of (4.14) is independent of $s$ while in the first member depends on it. In order to avoid this difficulty, we introduce a new function 9 defined by the formula

$$
\vartheta(r, t)=\int_{t}^{T} u_{\tau \tau} d \tau
$$


Then

$$
u_{t}(r, t)=\vartheta(r, s)-\vartheta(r, t) \quad \text { and } \quad u_{t}(r, T)=\vartheta(r, s) .
$$

Thus, inequality (4.14) can be written as

$$
\begin{aligned}
\left\|u_{t t}(r, s)\right\|_{L_{\rho}^{2}(0, R)}^{2} & +\left\|\mathfrak{J}_{r}\left(\rho u_{t t}(r, s)\right)\right\|_{L^{2}(0, R)}^{2}+(1-2 R(T-s))\left\|\vartheta_{r}(r, s)\right\|_{L^{2}(0, R)}^{2} \\
& \leq 2 R\left(\left\|\mathfrak{I}_{r}\left(\rho u_{t t}\right)\right\|_{L^{2}\left(Q_{s}\right)}^{2}+\left\|\vartheta_{r}\right\|_{L^{2}\left(Q_{s}\right)}^{2}\right) .
\end{aligned}
$$

If $s_{0}>0$ satisfies $2 R\left(T-s_{0}\right)=1 / 2$, then (4.17) implies that

$$
\begin{aligned}
\left\|u_{t t}(r, s)\right\|_{L_{\rho}^{2}(0, R)}^{2} & +\left\|\mathfrak{J}_{r}\left(\rho u_{t t}(r, s)\right)\right\|_{L^{2}(0, R)}^{2}+\left\|\vartheta_{r}(r, s)\right\|_{L^{2}(0, R)}^{2} \\
& \leq 4 R\left\|\mathfrak{J}_{r}\left(\rho u_{t t}\right)\right\|_{L^{2}\left(Q_{s}\right)}^{2}+\left\|\vartheta_{r}\right\|_{L^{2}\left(Q_{s}\right)}^{2}
\end{aligned}
$$

for all $s \in\left[T-s_{0}, T\right]$.

Making

$$
h(s)=\left\|\mathfrak{I}_{r}\left(\rho u_{t t}\right)\right\|_{L^{2}\left(Q_{s}\right)}^{2}+\left\|\vartheta_{r}\right\|_{L^{2}\left(Q_{S}\right)}^{2}
$$

in (4.18), we get

$$
\left\|u_{t t}(r, s)\right\|_{L_{\rho}^{2}(0, R)}^{2}-\frac{d h}{d s} \leq 4 R h(s),
$$

from which we have,

$$
-\frac{d}{d s}\left(h(s) e^{4 R s}\right) \leq 0 .
$$

Since $h(T)=0$, an integration of (4.21) with respect to $t$ over $[s, T]$ gives

$$
h(s) \cdot e^{4 R s} \leq 0 .
$$

It follows from inequality (4.22) that $\omega \equiv 0$ almost everywhere on $Q_{T-s_{0}}$. Since the length $s$ is independent of the origin, we use the same procedure a finite number of times to show that $\omega \equiv 0$ in $Q$. This completes the proof of Proposition 2.

Let $W=\left(\omega, \omega_{1}, \omega_{2}\right) \in R(L)^{\perp}$, such that

$$
(\mathscr{L} u, \omega)_{L_{\rho}^{2}(Q)}+\left(\ell_{1} u, \omega_{1}\right)_{V_{\rho}^{1}(0, R)}+\left(\ell_{2} u, \omega_{2}\right)_{L_{\rho}^{2}(0, R)}=0 .
$$

Putting $u \in D_{0}(L)$ into equation (4.23), we obtain

$$
(\mathscr{L} u, \omega)_{L_{\rho}^{2}(Q)}=0 \text { for all } u \text { in } D_{0}(L) .
$$

Hence, by virtue of Proposition 2, we deduce that $\omega \equiv 0$. Thus, equation (4.23) becomes

$$
\left(\ell_{1} u, \omega_{1}\right)_{V_{\rho}^{1}(0, R)}+\left(\ell_{2} u, w_{2}\right)_{L_{\rho}^{2}(0, R)}=0 .
$$

$\ell_{1} u$ and $\ell_{2} u$ are independent, and the ranges of the operators $\ell_{1}$ and $\ell_{2}$ are everywhere dense in the Hilbert spaces $V_{\rho}^{1}(0, R)$ and $L_{\rho}^{2}(0, R)$, respectively. Hence, $\omega_{1} \equiv 0$ and $\omega_{2} \equiv 0$. Consequently, $W \equiv 0$. This completes the proof of Theorem 2 . 


\section{REFERENCES}

[1] A. Bouziani, On a third order parabolic equation with a nonlocal boundary condition, to appear in J. Appl. Math. Stochastic Anal.

[2] _ Solution forte d'un problème mixte avec condition intégrale pour une classe d'équations heperboliques, Bull. CL. Sci., Acad. Roy. Belg. 8 (1997), 53-70.

[3] __ Solution forte d'un problème mixte avec condition intégrale pour une classe d'équations paraboliques, Magreb Math. Rev. 6 (1997), 1-17.

[4] _ Strong solution for a mixed problem with nonlocal condition for certain pluriparabolic equations, Hiroshima Math. J. 27 (1997), 373-390. Zbl 893.35061.

Mesloub: Département de Mathématiques, Centre Universitaire De Tebessa, 12000, ALGÉRIE

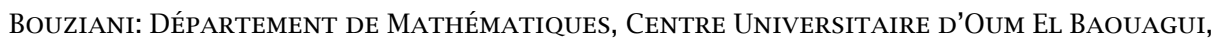
BP. 565, 04000, ALGÉRIE 


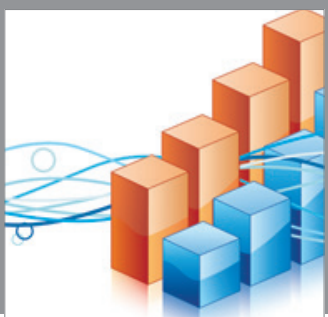

Advances in

Operations Research

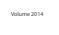

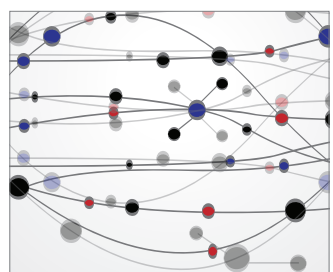

\section{The Scientific} World Journal
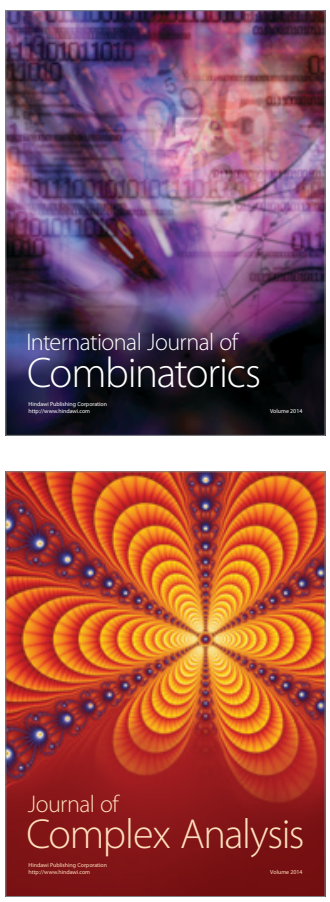

International Journal of

Mathematics and

Mathematical

Sciences
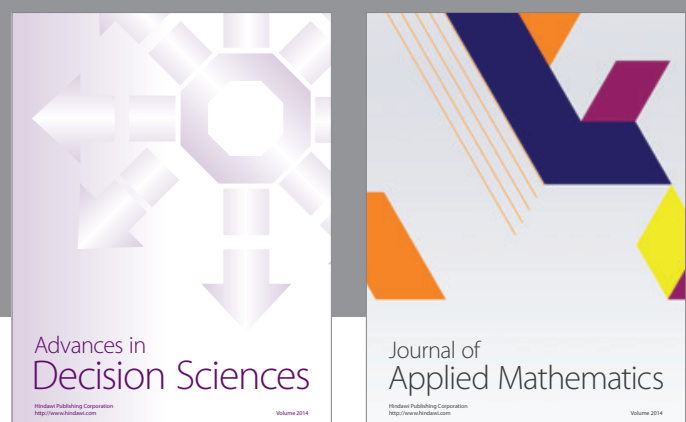

Journal of

Applied Mathematics
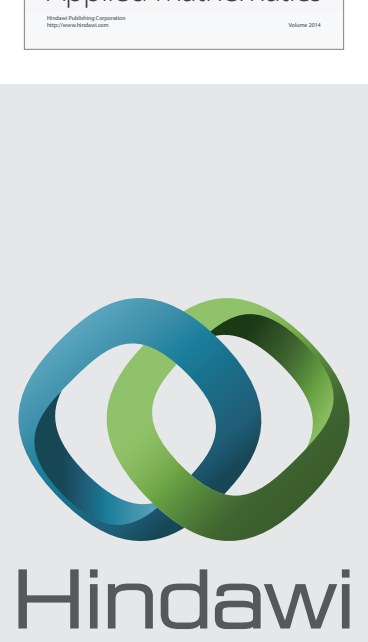

Submit your manuscripts at http://www.hindawi.com
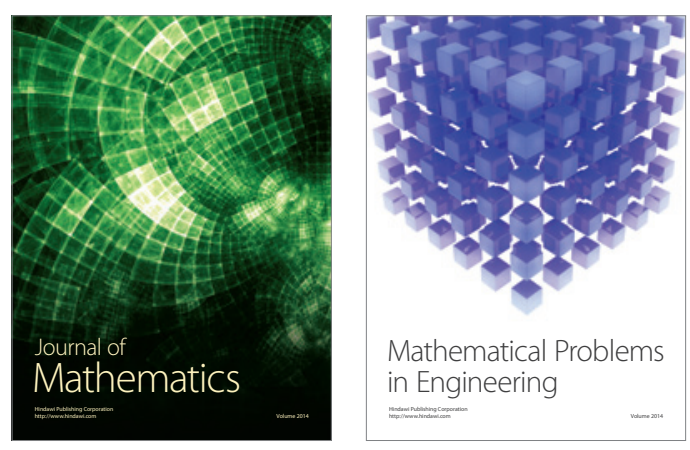

Mathematical Problems in Engineering
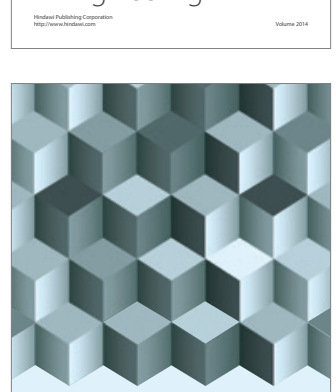

Journal of

Function Spaces
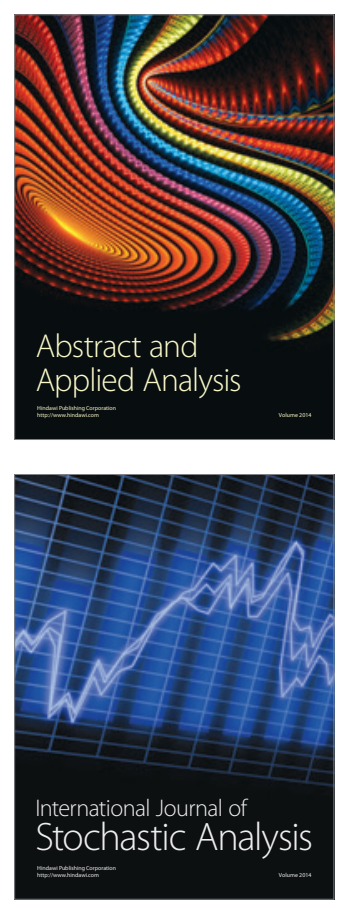

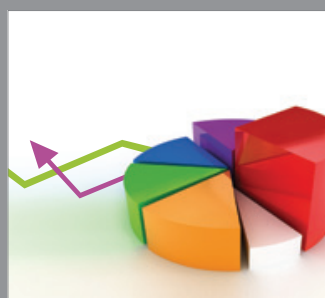

ournal of

Probability and Statistics

Promensencen
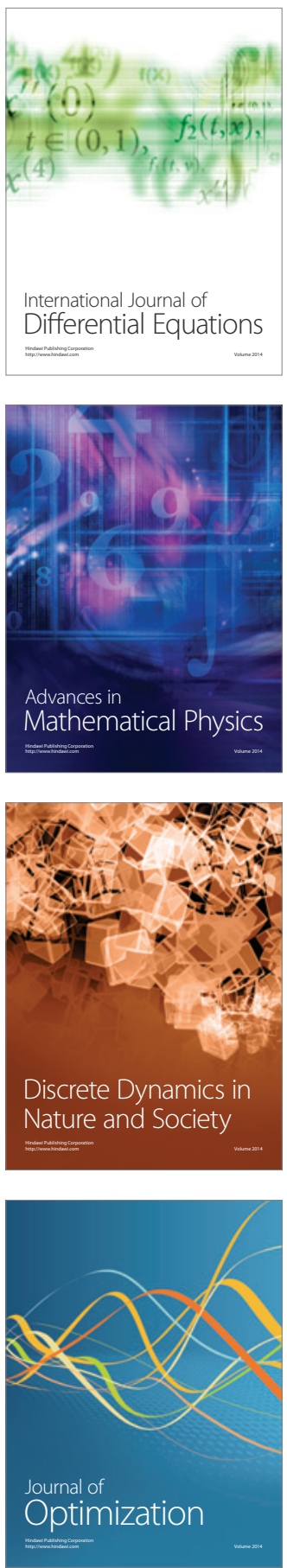